\title{
Paravertebral anesthesia and analgesia
}

Jonathan Richardson MD MRCP FRCA FIPP

$\mathrm{T}$

HE peripheral nerve blocking technique of paravertebral analgesia (PA) seems to remain the poor relation to central neural blocks. Although very high quality analgesia can be produced with very little cost in terms of side effects and complications, interest remains low and geographically sporadic. The reasons are ignorance, inertia and prejudice, but not science. The aim of publications such as this is to generate interest and dispel ignorance. There are three recent review articles to which the attention of the interested reader is drawn. ${ }^{1-3}$ All unreferenced statements are referenced in these. Many things about PA have yet to be worked out. Research opportunities are rich.

\section{Definition}

$\mathrm{PA}$ is defined as induction of anesthesia or analgesia through the deposition of local anesthetic (LA) alongside the vertebral column, close to the emergence of the spinal nerve.

\section{Anatomy and spread of injectate}

The "paravertebral space" (PVS) does not exist. It is a potential space which can be created by fluid distension. If a needle is placed alongside the vertebral column at or just anterior to the transverse process, but posterior to the parietal pleura and fluid (e.g., LA) is injected it will distend and open a wedge shaped space with the following boundaries:
TABLE I Boundaries of paravertebral space

\begin{tabular}{|c|c|}
\hline Posterior & $\begin{array}{l}\text { Superior costotransverse ligament. Laterally to that } \\
\text { the posterior intercostal membrane }\end{array}$ \\
\hline Anterior & Parietal pleura \\
\hline Medial & $\begin{array}{l}\text { Postero-lateral aspect of the vertebra, intervertebral } \\
\text { disc, intervertebral foramen }\end{array}$ \\
\hline Superior & Occiput \\
\hline Inferior & Alar of the sacrum \\
\hline Lateral & No limit, contiguous with the intercostal space \\
\hline
\end{tabular}

LA injected in this area will bathe the following neurological structures: the anterior and posterior ramus of the spinal nerve and the white and grey rami communicantes. In the thoracic region the sympathetic chain will be exposed to injectate as it is lateral to the vertebral body, not antero-lateral as in the lumbar region. In the lumbar region the sympathetic chain may not be involved as it is separated from more posterior structures arising from the intervertebral foramen by the origin of iliopsoas from the lateral vertebral bodies.

TABLE II Potential tracking of injectate from deposition with paravertebral space

\begin{tabular}{ll}
\hline Superior \& inferior & $\begin{array}{l}\text { Within the paravertebral gutter. In the } \\
\text { thorax across the heads \& necks of ribs } \\
\text { Mhrough the intervertebral foramen } \\
\text { (epidural anesthesia) }\end{array}$ \\
Lateral & $\begin{array}{l}\text { Contribution to cervical, stellate ganglion, } \\
\text { brachial plexus, intercostal \& lumbar plexus } \\
\text { blockade }\end{array}$ \\
Anterior & Not possible unless pleura breached
\end{tabular}

\section{Nomenclature}

PA involving the cervical region is better known as a deep cervical plexus block or possibly a stellate ganglion block (which lies directly anterior to the nerve roots of C6 \& C7). Lumbar PA is known as a lumbar plexus block or a psoas compartment block. The sacral spine cannot be subjected to PA due to fusion of the transverse processes to form the lateral mass.

\section{The mechanism of action}

The mechanism of action of PA is by direct penetration of LA into the spinal nerve, including the dorsal ramus, the rami communicantes and the sympathetic chain. The spinal nerve as it emerges from the intervertebral foramen lacks both the epineurium and part of the perineurium of the peripheral nerve and has only a thin membranous root sheath. It is therefore

From the Department of Anaesthetics, Bradford Royal Infirmary, Bradford, England.

Address correspondence to: Dr. J. Richardson, Department of Anaesthetics, Bradford Royal Infirmary, Bradford BD9 6RJ, England.

Phone: 00441274 364066; Fax: 00441274 366548; E-mail: docjohnnyr@hotmail.com 
easily penetrated by LA. Mass movement of drugs across further tissue planes is unnecessary for analgesia. However, movement of LA away from the site of deposition, in any direction, will contribute to analgesia (not counting intravascular spread; Table II).

Transforaminal spread will produce a dense block of the spinal nerve. This technique is employed by interventional pain practitioners. Approximately $1 \mathrm{~mL}$ or less of LA deposited just inside the intervertebral foramen produces a very high density block of prolonged duration. ${ }^{4}$ The amount of LA which passes from the PVS to the epidural space is impossible to predict but the likely involved factors are listed in Table III.

TABLE III Factors increasing the likelihood of transforminal (epidural) spread

\begin{tabular}{ll}
\hline Needle position & $\begin{array}{l}\text { Proximity to the intervertebral foramen. } \\
\text { Needle advancement inferior (not superior) } \\
\text { to transverse process. } \\
\text { Bevel turned medially. } \\
\text { Pressure gradients } \\
\text { Large volume and high speed of injection } \\
\text { overcomes mean negative intrathoracic } \\
\text { pressure }\end{array}$ \\
& $\begin{array}{l}\text { Lack of foraminal stenosis, lateral disc } \\
\text { bulging, zygapophysial joint hypertrophy or } \\
\text { epidural fibrosis. }\end{array}$ \\
\hline
\end{tabular}

The overall contribution of a unilateral epidural block induced through paravertebral deposition cannot be stated with any certainty, but it is likely to be substantial. Bilateral epidural blockade is very unusual.

\section{Quality of analgesia}

An accompaniment of the somatic block is a unilateral sympathetic block. This is due to penetration of the sympathetic chain and the slender semi-myelinated rami communicantes by LA. In a study of chronic pain patients undergoing PA with $15 \mathrm{~mL}$ of $0.5 \%$ bupivacaine, a mean somatic block of 5 dermatomes was accompanied by a mean sympathetic block of 8 dermatomes, as evidenced by thermographic ipsilateral skin warming.

The quality of block at the dermatomal site of injection is very high. Somatosensory evoked potential studies suggest that the degree of afferent blockade is higher than that of spinal or epidural anesthesia. The reasons for this are interesting and are a matter of debate. It is the author's belief that a number of factors come into play. Bathing of the (relatively naked) spinal nerve in the intervertebral foramen mimics the technique of transforaminal sleeve nerve root blockade which his known in chronic pain work to produce very profound anesthesia of prolonged duration. ${ }^{4}$
Blockade of the rami communicantes as well as the sympathetic chain results in a different and higher quality of block compared to central neural blocks. With PA the afferent and efferent sympathetics are blocked, whereas with central neural blocks only the efferent sympathetics to the surgical site are reliably blocked. This is because within the sympathetic chain inputs travel for a number of dermatomes before entering the spinal cord. This means that with central neural blocks a nerve pathway may be completely unaffected by LA so that afferents can enter the neuroaxis above or below the central block.

\section{Indications ${ }^{\mathrm{l}-3}$}

Thoracic PA is particularly advocated for unilateral surgical procedures, e.g., thoracotomy, ${ }^{8,9}$ breast surgery, ${ }^{10-12}$ cholecystectomy, ${ }^{13}$ cardiac surgery, ${ }^{15}$ and renal or ureteric surgery. It can also provide analgesia for rib trauma and herniorrhaphy. ${ }^{16,17}$ It has been used as a bilateral technique in thoracic surgery and major abdominal vascular surgery. In chronic pain management, it is used for the treatment of benign or malignant neuralgia, in the management of complex regional pain syndromes with a sympathetic component and in the therapeutic control of hyperhydrosis.

Lumbar and cervical paravertebral blocks are used for lower limb, usually orthopedic surgery, ${ }^{18-25}$ and carotid endarterectomy respectively and in chronic pain management.

\section{Techniques}

Loss of resistance to air or saline can be used but has the drawback of an indistinct endpoint. The patient can be in the prone, lateral (usually with the side to be blocked uppermost) or sitting position. Two or three centimetres lateral to the spinous process, a Tuohy needle is advanced perpendicular to all skin planes. At approximately 2 to $5 \mathrm{~cm}$ in an adult, the transverse process should be contacted. The needle is then reangled superiorly or inferiorly and advanced 1 or 1.5 $\mathrm{cm}$ until loss of resistance to air or saline is appreciated. A "click" may be felt as the superior costotransverse ligament is penetrated. The depth from the skin is variable but in the thoracic region the PVS is usually at 4 to $6 \mathrm{~cm}$. Paresthesia may be experienced by an awake patient.

Compared to epidural space location where a very resistant ligamentum flavum is traversed, followed by almost free injection, thoracic PVS location by this method is indefinite as the superior costo-transverse ligament may not be distinct and paravertebral areolar tissue provides more resistance to injection of air or saline than epidural fat. 
A number of maneuvers have been produced to improve reliability. Greengrass and colleagues ${ }^{2}$ recommend abandonment of attempted loss of resistance in favour of simple advancement 1 or $1.5 \mathrm{~cm}$ caudally beyond initial contact with the transverse process. Aspiration is followed by injection with no attempt to find loss of resistance. Also, every dermatomal level required to be anesthetized is injected rather than a large bolus depot being used. There is much to commend this technique. It is simple, quick and safe. There is a low incidence of unblocked segments and if accidental $i v$ entry is gained, with the total dose divided between perhaps five injections (e.g., for breast surgery), its toxic effects are unlikely to be severe.

There are additional ways of improving reliability. $X$-ray screening with contrast injection or continuous monitoring of needle tip pressures in spontaneously breathing patients can distinguish PVS from im or intrapleural space $^{5}$ and nerve stimulation demonstrates proximity to the appropriate nerve. ${ }^{11}$

Catheterization is straightforward but requires some manipulation of the needle bevel and firmness of advancement compared with epidural catherization. Advancement into the PVS superiorly over the top of the transverse process makes catheter feeding easier than if the needle is advanced inferior as the needle/catheter angle is less acute.

Direct vision feeding of catheters either at open thoracotomy or with video assisted thoracic surgery is very reliable and satisfactory, even in the smallest of infants.

\section{Drugs and doses}

Any LA can be used. Bupivacaine and lidocaine, both with and without adrenaline have been most studied. A bolus of $20 \mathrm{~mL}$ of bupivacaine $0.5 \%$ in the adult produces acceptable blood levels. Infants with a median age range of 5.3 weeks given a bolus followed by infusion of bupivacaine $0.25 \%$ for post-thoracotomy pain relief can also have satisfactory peak loading dose and infusion levels. This is also true for even smaller infants given bupivacaine $0.125 \%$.

The addition of adrenaline 1:200 000 lowers blood levels. Lidocaine with adrenaline by infusion in children reaches a steady state by eight to ten hours and is not associated with symptoms or signs of toxicity. To my knowledge there have been no reports of severe central nervous system or cardiovascular system toxicity either due to excessive systemic LA levels or due to profound sympathetic blockade.

Combinations of LA with adjuncts such as opiates and clonidine may also be very helpful in improving the block quality and duration. ${ }^{11,17}$
The dose of LA required involves a consideration of the number of dermatomes which it is desired to block. No reliable formula has as yet been developed, but it can be assumed in an adult that a volume of $15 \mathrm{~mL}$ will spread over and block at least 3 dermatomes and in children a bolus dose of $0.5 \mathrm{~mL} \cdot \mathrm{kg}^{-1}$ will reliably cover at least 4 dermatomes. In a recent study we failed to set up a definitive relationship of volume with single bolus $v s$ number of dermatomes blocked. ${ }^{26}$ For this reason, as well as others, a multiple level block technique in the thoracic region should be used instead of using a large bolus at the central dermatome.

In adult thoracotomy pain management, we use plain bupivacaine with a concentration ranging from 0.25 to $0.5 \%$, starting with a bolus dose of 15 to 20 $\mathrm{mL}$, at least ten minutes prior to skin incision. This bolus would be divided among probably 6 dermatomes through a multiple injection technique. A further bolus of $10 \mathrm{~mL}$ bupivacaine $0.5 \%$ is given during chest closure and this is followed by an infusion of bupivacaine $0.5 \%$ at a rate of $0.1 \mathrm{~mL} \cdot \mathrm{kg}^{-1} \cdot \mathrm{hr}^{-1}$ for two days, after which the concentration is lowered to $0.25 \%$ for a further three days. Other authors agree that continuous infusion provides better analgesia than intermittent bolus doses.

\section{Postoperative care}

No additional nursing skills or observations are necessary other than those required for the routine care of postoperative patients. For continuous infusions an aseptic technique is required in changing syringes and an in-line bacterial filter is advocated. There is no need to look specifically for cardiovascular changes and mobilization should be encouraged. Analgesic regime based admission to high dependency care is unnecessary.

\section{Efficacy}

A full discourse on the efficacy of PA is beyond the space allowed for this article.

Postoperative PA can provide high quality pain relief, as shown by low pain scores and opiate sparing. Sufficient analgesia in some situations, particularly breast surgery, has obviated the need for general anesthesia ${ }^{1-3,10,11,17}$ In minor breast surgery, although better pain relief is provided by PA compared to general anesthesia, the risk/benefit ratio has been found not to be routinely favourable. ${ }^{12}$

Thoracotomy pain is severe and is associated with major impairment of pulmonary function. Clear advantages of PA have been demonstrated in regard to both of these aspects compared to opiates, intrapleural bupivacaine or epidural bupivacaine. Postoperative pulmonary complications and hospital stays are 
reduced, as is the generation of chronic pain (postthoracotomy neuralgia). Some aspects of stress inhibition have been demonstrated in patients undergoing thoracotomy (and cholecystectomy), effects which cannot be demonstrated for these types of surgery with more central forms of afferent blockade.

In minimally invasive direct coronary artery bypass surgery PA was determined to be equivalent to epidural anesthesia but was safer and easier to carry out. ${ }^{15}$

Not all clinical trials have been favourable. Perttunen and colleagues ${ }^{9}$ carried out a comparison of extradural, paravertebral and intercostal blocks for post-thoracotomy pain. Similar levels of pain, opiate requirements, pulmonary function and adverse effects were found in all groups. The dosage levels however in the paravertebral group were inadequate for reasonable blockade.

In lower limb orthopedic surgery spinal anesthesia combined with psoas compartment block was found to be superior to general anesthesia in terms of resource utilization, patient satisfaction and postoperative analgesia. ${ }^{25}$ Most studies show favourable results $^{20,25}$ although some do not. ${ }^{22,23,26}$ Reduced blood loss with total hip arthroplasty has been shown. ${ }^{20}$ In hip replacement compared with epidural blocks, similar analgesia has been found, but with lower motor blockade times, shorter ambulation time and significantly less complications. ${ }^{19}$

With inguinal herniorrhaphy in a prospective randomized trial PA was found to be superior to general anesthesia and spinal anesthesia in terms of postoperative nausea and vomiting, hospital stay and the need for postoperative analgesics. ${ }^{17}$

Bigler and colleagues ${ }^{13}$ compared epidural bupivacaine and morphine with paravertebral bupivacaine in post-cholecystectomy patients. Pain scores were significantly better in the epidural group compared to the paravertebral group. There was no difference in their effects on pulmonary function.

\section{Contraindications}

PA can be safely performed in anesthetized patients. A coagulation disorder or the use of anticoagulants is a relative rather than an absolute contraindication as the neurological consequences of a paravertebral hematoma are negligible. Occupation of the PVS by tumour is a contraindication, as is local sepsis at the proposed site. Pus between the pleurae (empyema) should not theoretically be disturbed by paravertebral cannulation but the accompanying acidosis would probably adversely effect LA ionization (and hence nerve penetration). This and the accompanying hyperemia would encourage systemic LA toxicity. However septic patients cannot safely be offered epidural anesthesia and hence, with caution, possibly it is advisable to proceed with PA.

Great care is needed in the case of severe chest deformity or scoliosis to avoid injection into the meninges or pleura.

A planned pleurectomy is not a contraindication. As long as the parietal pleura covering the vertebral bodies and a few centimetres distally is left intact, catheterization and a satisfactory block can be achieved.

\section{Side effects and complications}

PA generally has a low incidence of adverse effects. In retrospectively reviewing this subject, the overall incidence of side effects or complications was less than $5 \%$. In a multi-centred prospective study of 367 pediatric and adult patients the overall failure rate was found to be $10.1 \%$. The frequency of complications was: hypotension $4.6 \%$; vascular puncture $3.8 \%$; pleural puncture $1.1 \%$; and pneumothorax $0.5 \%$.

Neurological or hemodynamic events from accidental epidural or intrathecal entry have not been associated with any long term morbidity or mortality, even following very occasional reports of total spinal anesthesia. A Brown Séquard paralysis followed the non- $x$-ray guided use of paravertebral alcohol in $1931 .{ }^{27}$

Hypotension is unusual. A study of thoracic paravertebral blocks using $15 \mathrm{~mL}$ bupivacaine $0.5 \%$ produced a mean unilateral (thermographic) sympathetic block of 8 dermatomes but without any significant change in supine and sitting blood pressures. The occurrence of hypotension in $4.6 \%$ is multifactorial, usually involving unmasking of relative hypovolemia by unilateral vasodilatation.

\section{Discussion}

Excellence in anesthesia and perioperative medicine demands the use of regional anesthesia and analgesia in any situation where pain and the neuroendocrine stress response to trauma makes significant demands upon organ systems. This may be defined by the type of surgery, but it also depends upon the overall condition of the patient. Failing organ systems would sway the decision to employ regional nerve blocks towards more minor procedures. The type of block and when to apply it requires maturity of judgement. There is no scientific reason to exclude PA from one's armamentarium or indeed for not using it as the method of choice in many situations, particularly unilateral surgery. Published data strongly indicate that it is effective and safe. PA is simple to perform, has few 
contraindications and requires no additional postoperative care. It is applicable to large numbers of patients and because of its low side-effect profile (especially hypotension), it can form the backbone of an accelerated postoperative mobilization regime.

More studies are needed to define the mode of action, the incidence and contribution of epidural spread to analgesia. This whole subject is intriguingly open to reinterpretation in the light of new studies. Emerging data overall continue to lend support to the use of PA rather than bringing to light any previously unsuspected drawbacks.

\section{References}

1 Richardson J, Sabanathan S. Thoracic paravertebral analgesia. A review. Acta Anaesthesiol Scand 1995; 39: 1005-15.

2 Richardson J, Lönnqvist PA. Thoracic paravertebral blockade. A review. Brit J Anaesth 1998; 81: 230-8.

3 Karmaker MK. Thoracic paravertebral block. Anesthesiol 2001; 95: 771-80.

4 Riew KD, Yin Y, Gilula L, Birdwell KH, Lenke LG, Goette $K$. Can nerve root injections obviate the need for operative treatment for lumbar radicular pain? A prospective randomised, controlled double-blinded study. NASS Proceedings; Annual Meeting 1999.

5 Richardson J, Cheema SP, Hawkins J, Sabanathan S. Paravertebral space location: a new method using pressure measurement. Anaesthesia 1996; 51: 137-9.

6 Igarashi T, Hirabayashi $\Upsilon$, Shimizu R, Saitoh K, Fukuda $H$. Thoracic and lumbar extradural structure examined by extraduroscope. Br J Anaesth 1998; 81: 121-5.

7 Richardson J, McGurgan P, Cheema S, Prashad R. Gupta $S$. Spinal endoscopy in chronic low-back pain with radiculopathy. A prospective case series.

Anaesthesia 2001; 56: 454-60.

8 Caretta A, Zanini P, Chiesa G, Altese R, Melloni G, Grossi $A$. Efficacy of ketorolac tromethamine and extrapleural intercostals nerve block on post-thoracotomy pain. A prospective, randomised study. Int Surg 1996; 81: 224-8.

9 Perttunen K, Nilsson E, Heinonen J, Hirvisalo EL, Salo $J A$, Kalso E. Extradural paravertebral and intercostal nerve blocks for post-thoracotomy pain. Br J Anaesth 1995; 75: 541-7.

10 Pusch F, Freitag H, Weinstabl C, Obwegeser R, Huber E, Wilding $E$. Single injection paravertebral block compared to general anaesthesia in breast surgery. Acta Anaesthesiol Scand 1999; 43: 770-4.

11 Naja MZ, Ziade MF, Lönnqvist PA. Nerve-stimulator guided paravertebral blockade vs general anaesthesia for breast surgery: a prospective randomised trial. Eur J
Anaesthesiol 2003; 20: 897-903.

12 Terheggen MA, Wille F, Borel-Rinkes IH, Ionescu TI, Knape JT. Paravertebral blockade for minor breast surgery. Anesth Analg 2002; 94: 355-9.

13 Bigler D, Dirkes W, Hansen J, Rosenberg J, Keblet H. Effects of thoracic paravertebral block with bupivacaine versus combined thoracic epidural block with bupivacaine and morphine on pain and pulmonary function after cholecystectomy. Acta Anaesthesiol Scand 1989; 33: 561-4.

14 Ganapathy S, Murkin JM, Boyd DW, Dobkowski W, Morgan J. Continuous percutaneous paravertebral block for minimally invasive cardiac surgery. J Cardiothorac Vasc Anesth 1999; 13: 594-6.

15 Dhole S, Mehta $\Upsilon$, Sexana H, Juneja R, Trehan N. Comparison of continuous thoracic epidural and paravetertebral blocks for postoperative analgesia after minimally invasive direct coronary artery bypass surgery. J Cardiothorac Vasc Anesth 2001; 15: 288-92.

16 Klein SM, Pietrobon R, Nielsen KC, et al. Paravertebral somatic nerve block compared with peripheral nerve blocks for out-patient inguinal herniorrhaphy. Reg Anesth Pain Med 2002; 27: 476-80.

17 Naja MZ, el Hassan MJ, Oweidat M, Zbido R, Ziade $M F$, Lönnqvist PA. Paravertebral blockade vs general anaesthesia or spinal anesthesia for inguinal hernia repair. Middle East J Anesthesiol 2001; 16: 201-10.

18 Farny J, Girard M, Drolet P. Posterior approach to the lumbar plexus combined with sciatic nerve block using lidocaine. Can J Anaesth 1994; 41: 486-91.

19 Türker G, Uckunkaya N, Yavascaoglu B, Yilmazlar A, Ozcelik $S$. Comparison of the catheter technique psoas compartment block and the epidural block for analgesia after partial hip replacement surgery. Acta Anaesthesiol Scand 2003; 47: 30-6.

20 Stevens RD, Van Gessel E, Flory N, Fourner R, Gamulin $Z$. Lumbar plexus block reduces pain and blood loss associated with total hip arthroplasty. Anesthesiology 2001; 93: 115-21.

21 Capdevila X, Macaire P, Dadure C, et al. Contunous psoas compartment block for postoperative analgesia after total hip arthroplasty: new landmarks, technical guideleines and clinical evaluation. Anesth Analg 2002; 94: 1606-13.

22 Bogoch ER, Henke M, Mackenzie T, Olschewski E, Mabomed NN. Lumbar paravertebral nerve block in the management of pain after total hip and knee arthroplasty: a randomised controlled clinical trial. J Arthroplasty 2002; 17: 298-401.

23 Souron V, Delaunay L, Schifrine P. Intrathecal morphine provides better postoperative analgesia than psoas compartment block after primary hip arthroplasty. Can J Anesth 2003; 50: 574-9. 
24 Chelly JE, Casati A, Al Samsam T, Coupe K, Criswell $A$, Tucker J. Continuous lumbar plexus block for acute postoperative pain management after open reduction and internal fixation of acetabular fractures. J Orthop Trauma 2003; 17: 362-7.

25 Jankowski CJ, Hebl JR, Stuart MJ, et al. A comparison of psoas compartment block and spinal and general anesthesia for outpatient knee arthroscopy. Anesth Analg 2003; 97: 1003-9.

26 Cheema S, Richardson J, McGurgan P. Factors affecting the spread of bupivacaine in the adult paravertebral space. Anaesthesia 2003; 58: 684-711.

27 Molitch M, Wilson G. Brown-Séquard paralysis following a paravertebral alcohol injection for angina pectoris. JAMA 1931; 97: 247. 